\title{
LA ECONOMÍA CONDUCTUAL Y EL ANÁLISIS EXPERIMENTAL DEL COMPORTAMIENTO DE CONSUMO
}

\author{
Behavioral economics and the experimental analysis of costume behavior \\ William Montgomery U. ${ }^{1}$ \\ Universidad Nacional Mayor de San Marcos, Lima, Perú \\ (Recibido el 15/04/2011 - Aceptado el 08/06/2011)
}

\begin{abstract}
RESUMEN
El presente artículo considera el aporte del análisis experimental del comportamiento en el marco del desarrollo de la economía conductual, y su complementariedad con las investigaciones actuales en psicología económica. En primer lugar, se hace una revisión de los orígenes y desarrollo de la economía conductual desde los principios básicos que fundamentan la ley de utilidad marginal en relación con los estados de privación y saciedad del comportamiento, hasta el papel de la economía de fichas y la regulación conductual. En segundo lugar, se discuten los estudios sobre el tema de la relación entre la economía conductual y los procesos decisorios del comportamiento de consumo, concluyendo que demuestran ser de gran utilidad conceptual para evaluar innumerables situaciones aplicadas.
\end{abstract}

Palabras clave: Economía conductual, análisis experimental del comportamiento, regulación conductual, economía de fichas, comportamiento de consumo, psicología económica.

\begin{abstract}
This paper considers the contribution of the experimental analysis of behavior in the context of development of behavioral economics, and its complementarity with current research in economic psychology. First, we review the origins and development of behavioral economics from the basic principles underlying the law of marginal utility in relation to states of deprivation and satiation behavior, the role of the token economy and regulation behavior. Secondly, we discuss studies on the subject of the relationship between behavioral economics and decision-making consumer behavior, concluding that prove useful for evaluating conceptual countless situations applied.
\end{abstract}

Keywords: Behavioral economics, experimental analysis of behavior, behavior regulation, token economy, consumer behavior, economic psychology.

1 Docente Asociado de la Facultad de Psicología de la Universidad Nacional Mayor de San Marcos. E-mail: avidolector@yahoo.es 


\section{INTRODUCCIÓN}

La Psicología Económica pretende una integración interdisciplinar de Economía y Psicología, lo que se estudia principalmente es la forma cómo los seres humanos adquieren bienes y servicios, toman decisiones, ahorran, compran, se endeudan, y pagan o evaden sus impuestos (Denegri, 2004/2010; Alejo, Rojas y Pérez-Acosta, 2008). En este contexto, la etiqueta de "economía conductual" o "economía del comportamiento" se ha hecho familiar para muchos economistas y psicólogos. Inclusive a uno de los propulsores de su versión más conocida, la teoría de las perspectivas, el Dr. Daniel Kahneman, se le concedió el Premio Nobel de Economía en el año 2002.

Dicha teoría, al igual que las tendencias interdisciplinares de las llamadas "finanzas conductuales" y la "economía experimental" (Vieira, 2008), se halla más implicada en los estudios de psicología cognitiva, pero, como reseñan Francisco, Madden y Barrero (2009), hay otra connotación, también interdisciplinaria pero menos conocida (aunque más representativa), de economía conductual ligada estrictamente al uso de los principios, procedimientos y aplicaciones relacionadas con el análisis experimental del comportamiento. Esta modalidad es frecuentemente pasada por alto en los libros de texto, artículos y reportes de divulgación de la psicología económica (por ejemplo, la reciente revisión hecha para la Fundación Health and Safety Executive de Sapford, Phythian-Adams y Apps, 2009), pese a mostrar un gran dinamismo en el planteamiento y realización de investigaciones científicas, en especial respecto al comportamiento del consumidor.

Por ello, el propósito de este artículo es considerar el aporte del análisis experimental del comportamiento en el marco del desarrollo de la economía conductual, y su complementariedad con la presente ola de investigaciones en psicología económica. En este contexto, como dicen Mayorga, Albañil y Cómbita (2006): "La economía conductual es una teoría del refuerzo que permite explicar diferentes comportamientos tanto animales como humanos enmarcados en la conducta de elección, donde los organismos toman decisiones a partir de las condiciones ambientales que se encuentran establecidas en un momento dado" (p. 34).

El plan del trabajo es, primero, revisar los orígenes y desarrollo de la economía conductual desde los principios básicos que fundamentan la ley de utilidad marginal en relación con los estados de privación y saciedad del comportamiento, hasta el papel de la economía de fichas y la regulación conductual. Luego se discuten los estudios sobre el tema de la relación entre la economía conductual y los procesos decisorios del comportamiento de consumo.

\section{La economía conductual desarrollada desde el análisis conductual}

\section{Oferta y demanda, privación y saciedad}

Alejo, Rojas y Pérez-Acosta (2008) refieren que, para fuentes importantes, el recuento histórico de la psicología económica se debe comenzar a partir de la escuela marginalista, en relación con la obra Fundamentos de la Economía Nacional del pensador austríaco 
Karl Menger en 1871. Él, junto con otros defensores del concepto de utilidad marginal (Böhm-Barew, Von Weiser, Walras, Jevons), sostuvo la validez universal de las leyes clásicas e introdujo en el análisis económico la teoría subjetiva del valor.

Sin embargo, es conveniente señalar que para los filósofos del utilitarismo del siglo XVIII y XIX, ya estaban claros los principios implicados en el cambio de valor de objetos y eventos como resultado de variaciones relativas a su accesibilidad o esfuerzo para producirlos. Por ejemplo, Bentham consideraba la riqueza y la felicidad o bienestar individuales como beneficios correlacionados. Mill, por su parte, enunció la ley de la oferta y la demanda en términos inequívocos: "La oferta y la demanda... serán iguales. Si son desiguales en algún momento, la competencia las iguala y la forma en que se hace es ajustada al valor. Si la demanda aumenta, el valor se eleva, si la demanda disminuye, el valor baja; asimismo, si la oferta cae, el valor se eleva y cae si la oferta se aumenta..." (Cit. por Staats, 1975/1979; pp. 445-446). De esta manera, mientras más cantidad tenga una persona de un artículo de consumo en un momento dado, menos encontrará utilidad o satisfacción en tener más de lo mismo; y por lo tanto reajustará su presupuesto para no obtenerlo. Y lo opuesto: mientras menos tenga de algún producto, más utilidad o satisfacción encontrará en poseerlo, dirigiendo la actividad hacia su adquisición. Así, el precio de un bien queda establecido a través de su utilidad marginal, no a través de la utilidad objetiva. En el famoso ejemplo de Bentham (ed. 1978) sobre el agua y los diamantes, éste pensador sostiene que donde el agua es abundante su utilidad marginal es baja; mientras que la de los diamantes es alta por su poca disponibilidad. Si fuera al revés, sería el agua la que tuviera la utilidad marginal más alta.

Es evidente que la ley económica de la oferta y la demanda sobre los consumidores resulta ser una expresión relativamente cercana a los efectos de la saciedad y la privación en el análisis experimental conductual. En los experimentos sobre saciedad, ésta es definida como una operación que consiste en presentarle continuamente un reforzador natural (alimento) a un organismo (por ejemplo una rata o una paloma, hasta que la tasa de respuestas se reduce: deja de comer). A su vez, la privación consiste en retirarle al organismo el reforzador natural durante cierto tiempo, dando por resultado en el proceso la elevación de la tasa de respuestas (apretar una palanca o picotear un disco) para conseguirlo. Estos procesos podrían enunciarse en la forma de principios:

\begin{abstract}
Saciedad
"Si un reforzador se presenta continuamente durante algún tiempo, la tasa de respuestas
\end{abstract}

asociada al reforzador tenderá a reducirse”.

Privación

"Si se retira un reforzador durante algún tiempo, la tasa de respuestas asociada a la aproximación al reforzador tenderá a elevarse”. 
Como es fácilmente deducible, los principios en mención tienen que ver respectivamente: a) en el caso de la saciedad, con el exceso de oferta que reduce la demanda (baja utilidad marginal), y, b) en el caso de la privación, con la carencia de oferta que eleva la demanda (alta utilidad marginal), durante un período determinado.

El reconocimiento de esta relativa equivalencia impulsa los estudios básicos de investigación desde el análisis experimental del comportamiento. A este respecto, son de relevancia los hallazgos obtenidos a partir de las investigaciones sobre la economía de fichas y sobre la hipótesis de la regulación conductual.

\section{El papel de la economía de fichas}

En un comienzo, la integración de los conceptos económicos con los estudios de laboratorio sobre el sistema de fichas proporcionó hipótesis muy sugerentes (Staats y Staats, 1963), que fueron sometidas a contrastación empírica en varios estudios (Staats, Staats, Schutz y Wolf, 1962; Ayllon y Azrin, 1965; Phillips, Phillips, Fixsen y Wolf, 1971; Winkler, 1971), llegándose a conclusiones tales como que la paga afecta la oferta de trabajo, que hay una relación entre el ahorro y la cantidad de trabajo (a mayor ahorro, menos trabajo), y que dicha relación podía modificarse introduciendo variaciones en la gama de bienes ofrecidos u otorgando "intereses".

Estos resultados alentaron a Kagel y Winkler (1972) a proponer explícitamente la integración interdisciplinaria entre la economía y los principios del reforzamiento. Ellos vieron que los sistemas de fichas, tal como se utilizaban en la modificación de conducta de poblaciones de salas hospitalarias, podían ser verdaderos laboratorios de observación controlada sobre los intercambios de unidades con el comportamiento de los sujetos, pues tenían mucho que ver con sistemas económicos. Allí se podían entender las fichas como equivalentes a dinero, las distribuciones de fichas en plan de reforzadores condicionados como pagos salariales, y las tasas de cambio de las fichas por reforzadores como precios de bienes consumidos. Eso proporcionó la observación de variadas relaciones entre los ingresos y los patrones de consumo en los programas de razón, además de certificar la relevancia de los conceptos económicos básicos (como por ejemplo la elasticidad de la demanda) para la economía de fichas, y la importancia de todo eso para el arsenal terapéutico (Fisher, Winkler, Krasner, Kagel, Battaliod \& Basmann, 1978). El caso es que estos primeros escarceos con fichas fundaron las bases de la economía conductual operante.

\section{La regulación conductual}

Como señala Domjan (2007/2010), los estudios experimentales se han centrado en los últimos tiempos sobre la hipótesis de la regulación conductual y su llamado "punto de éxtasis" (o de estabilidad en la que el organismo se siente cómodo), lo que constituye un tercer factor de desarrollo gracias al cual se deben los avances experimentales en la investigación sobre economía del comportamiento.

La hipótesis de la regulación conductual opera sobre la suposición de que todo organismo actúa con una pauta determinada de distribución de sus respuestas, las cuales tiende a 
preferir o mantener en el mismo nivel pese a interrupciones o turbulencias. Por ejemplo, las actividades que implican supervivencia (comer, beber, abrigarse, ejercitarse, etc.) tienen un punto particular de "comodidad" o "bienestar" en el que la tasa de conducta de cada organismo requiere estar para experimentarlo, ni más ni menos. Eso es el "punto de éxtasis conductual", identificable mediante la frecuencia relativa de distribución de las respuestas de un organismo en situaciones no restrictivas, o sea, durante un período de línea base de operante libre en que se observan las elecciones espontáneas que hace el individuo, de acuerdo con sus disposiciones personales.

Llevando esto a una situación cotidiana, encontraríamos que si una persona con vocación deportiva y poco interés noticioso tiene las opciones de hacer ejercicios aeróbicos durante una hora o ir a ver noticieros televisivos, preferirá estadísticamente lo primero si no hay restricciones a esa conducta. ¿Qué pasaría si se introduce durante cierto período la condición de que dicha persona sólo puede tener libertad para hacer los ejercicios durante diez minutos siempre y cuando mire antes otros diez minutos constantes de televisión, y así sucesivamente? La hipótesis señala que el individuo del ejemplo intentaría volver a su "punto de éxtasis" (la cantidad de ejercicio que requiere) reasignando su conducta tan pronto como la contingencia impuesta se lo permitiera. Esto puede parecer contradictorio dadas las restricciones, pero debe tenerse en cuenta que esta relación entre respuesta instrumental y respuesta reforzante se enmarca en un contexto más amplio, que brinda las posibilidades de reasignación conductual. Por ejemplo, puede ser que la persona en cuestión utilice algún "sustituto momentáneamente aceptable" del ejercicio aeróbico durante el tiempo que ve las noticias por televisión: respirar acompasado, agitar piernas y brazos, hacer flexiones apoyándose en el asiento, o algo así.

Ese contexto amplio de opciones de respuesta tiene una semejanza grande con el ámbito de las elecciones de consumo en el campo económico. De este modo, se constituye en un área investigativa interdisciplinaria, ayudando a identificar la distribución de las respuestas de un individuo en un marco sistémico de limitaciones que afectan su asignación, tal como sucede en los procesos de utilidad marginal (Allison, 1993; Bickel, Green y Vuchinich, 1995).

\section{Economía conductual y procesos decisorios}

\section{Regulación conductual del comportamiento del consumidor}

Las restricciones económicas en una situación de mercado se entienden como los montos de ingreso y precio de los bienes que se quieren adquirir. Para la hipótesis de la regulación conductual, al manipular el programa de reforzamiento el experimentador funge de proveedor que fija el precio de un producto ofrecido en el mercado. En este encuadre conceptual, el precio es definido en los experimentos (igual que en la economía) como una relación costo-beneficio denominada precio unitario. El precio unitario de un bien especifica el precio pagado por una unidad estándar de refuerzo y se puede aumentar por incremento del costo (por ejemplo, las presiones de palanca para obtener refuerzo) o disminuyendo el beneficio (por ejemplo, la magnitud del refuerzo). Así, el monto de 
ingreso se traduce en el número de respuestas que un organismo puede emitir, y el precio de los bienes se traduce en la cantidad de respuestas requeridas para obtener un reforzador (Hursh y Silberberg, 2008).

En este contexto, un concepto fundamental es el de curva de demanda, o relación entre el precio de un bien y la cantidad de dicho bien. Por ejemplo, si el precio del producto aumenta significativamente, disminuirá con rapidez la cantidad comprada. No obstante, algunos bienes son más resistentes que otros a variar su consumo aunque se incremente su precio, debido a que existen ciertos determinantes contextuales de su poca elasticidad. La "elasticidad de la demanda" de un producto no es más que el grado alto o bajo en que el precio influye sobre su consumo. Si impacta poco se dice que la elasticidad de la demanda del producto es baja, y viceversa.

Tales determinantes vienen a ser las restricciones a que está sujeto el comportamiento del consumidor. Entonces, si alguien se pregunta cuáles son las posibilidades de la distribución de respuestas de un organismo en una contingencia abierta (es decir cómo una persona busca "atajos heurísticos" en "entornos de incertidumbre", en la terminología de los teóricos de la perspectiva), tiene que considerar el interjuego de todos esos tipos de restricción como variable determinante, que, en general, son tres.

El primero de ellos es la "disponibilidad de sustitutos". Los "sustitutos" son los reforzadores alternativos al reforzador central, cuya disponibilidad ocasiona que los incrementos en el número de respuestas que se necesitan para su demanda disminuyan. Esto distorsiona el grado en que los precios influyen en el mercado debido a que hay ciertos bienes muy sensibles a los cambios de precio y hay otros que no. Y eso es porque los primeros carecen de sustitutos. El ejemplo clásico de poca elasticidad es el del combustible, pues aunque suba mucho, su insustituibilidad es tal que la gente seguirá comprándolo. Caso distinto es el de las golosinas, cuya elasticidad es grande porque si varía mucho el rango de su precio la gente comprará menos.

En otro ejemplo, si en determinado momento se hace difícil conseguir alimentos "sanos" de cierta marca pero se dispone de otros más accesibles que ofrezcan una razonable cuota de reemplazo aunque no sean lo mismo, habrá mayor probabilidad de que un individuo se incline por las alternativas a ese consumo. Según las investigaciones (por ejemplo ver Murphy, Correla y Barnett, 2007), la fórmula es de triple consideración: las decisiones de los consumidores de un bien están relacionadas tanto con: a) su disponibilidad relativa y el precio del mismo, como con b) su disponibilidad relativa y el costo de actividades alternativas sin el producto, y c) el grado en que el reforzamiento de las consecuencias inmediatas se devalúa paralelamente a la acción del reforzamiento inmediato ligado al disfrute del bien.

En este contexto, el estudio de Mayorga, Albañil y Cómbita (2006) verifica si los sujetos experimentales sustituyen bienes como el alimento líquido en vez del alcohol, en la medida en que se aumenta el "precio" del alimento. Los resultados apoyan la hipótesis, tal como se ve en la Figura 1, que representa el programa experimental de refuerzo donde queda claro cómo al aumentar el "precio" de la sacarina, las ratas la sustituyen por el etanol. 


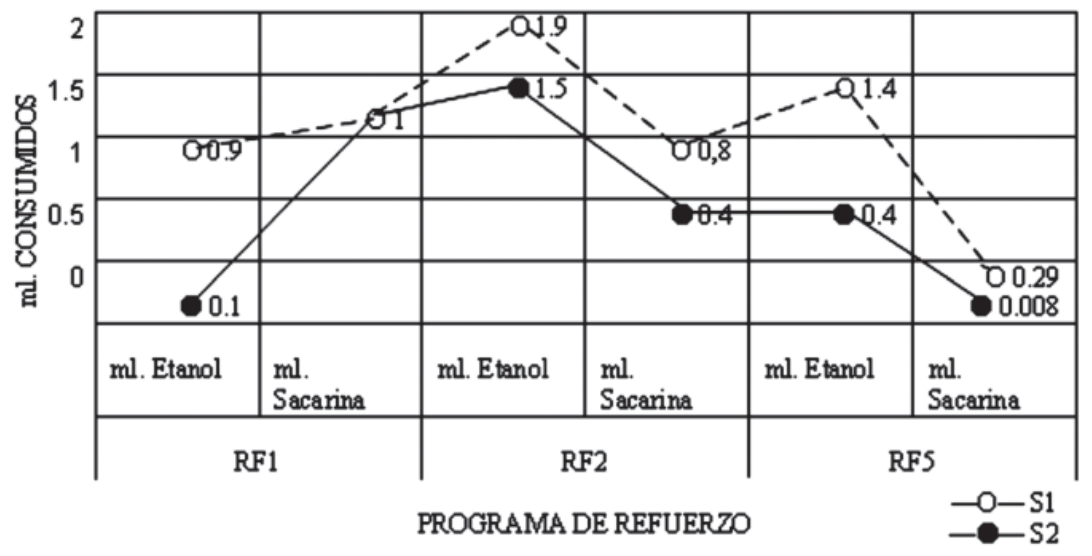

FIGURA 1. Comparación del consumo en los programas de refuerzo

Sujeto 1advs Sujeto 2

El segundo factor importante para la elasticidad y las restricciones mencionadas es el "rango de precios" del producto. Dicho rango está dado por la oscilación de los valores mayor y menor de costo, en el sentido de que si el precio de un producto siendo bajo se eleva un tanto, es probable que no afecte la demanda. En cambio, si siendo alto se eleva, es probable que sí la afecte. En los experimentos respectivos (Sumpter, Temple, \& Foster, 2004), parece una regla que los sujetos sometidos a la poca variación de precios bajos se ajusten a tal cambio incrementando el número de respuestas suficientes (por ejemplo, la duración y la fuerza necesarias) para obtener el reforzador. La cantidad de respuestas requeridas representa la dificultad para conseguir semejante expedición. Si la dificultad se incrementa mucho, habrá una disminución notable del esfuerzo por obtenerlo. Si se incrementa ligeramente, el individuo tiende a "ajustarse" aumentando también el número de respuestas que necesita. Por algo Hursh y Silberberg (2008) indican que la sensibilidad a los cambios en los precios (elasticidad) es una excelente medida de la eficacia del refuerzo.

Esto, si se lleva al plano de la decisión económica, tendrá efectos parecidos. Por ejemplo, si se sube ligeramente el costo de un pan barato, eso no impedirá que el consumidor promedio continúe pidiendo el mismo número de unidades aunque tenga que pagar 10 o 20 centavos más. Pero si se sube el precio de un pan caro por más que sea en poca cantidad, el consumidor muy probablemente suspenderá o disminuirá su adquisición.

Por último, el tercer elemento de restricción se mueve en torno al "nivel de ingresos". Vale decir, el individuo con un nivel alto de ingresos no sentirá mucho las variaciones en el costo de los productos. De hecho, a mayor nivel de ingresos habrá mayor insensibilidad al cambio de precios. Los experimentos realizados asimilan el "nivel de ingresos" que tiene el organismo con la disponibilidad de sus respuestas o tiempo para emplearlas. En la investigación de Silberberg, Warren-Boulton, y Asano (1987) se utilizaron monos para someterlos a la condición experimental de escoger entre una bola de comida grande pero amarga, y otra pequeña. En condición de "bajos ingresos" (menos intervalo entre ensayos, 
cada 60 a 70 segundos), los sujetos prefirieron la pastilla grande pero amarga. Cuando se invirtió la condición a "altos ingresos" (reducción de los intervalos a 15 segundos), produjo un incremento en el consumo de las bolas pequeñas. En otra investigación (véase DeGrandpre, Bickel, Rizvi y Hughes, 1994) a siete sujetos humanos dependientes del cigarrillo se les ofrecieron dos refuerzos distintos: uno consistente en bocanadas de la marca habitual de cigarrillos ("propios") que solían fumar, y otro consistente en bocanadas de una marca menos preferida de cigarrillos con un contenido similar de nicotina ("otros"). Los "otros" costaban la quinta parte del precio de los "propios". Se constató que cuando el precio de los dos refuerzos se mantuvo constante, las elecciones optaron por lo más barato. Sin embargo, al variar el monto del ingreso hacia arriba, la proporción del consumo de bocanadas "propias" aumentó, mientras que el consumo de los "otros", menos costosos, disminuyó.

De hecho, si, por ejemplo, dos fumadores recién llegados a una comunidad se encuentran con que hay una baja densidad de puntos de venta de tabaco en su entorno cercano, pero sólo uno de ellos tiene la posibilidad de transportarse más fácil y rápidamente hacia puntos lejanos para adquirir más cigarrillos, es de esperar que el que carezca de esa facilidad baje su consumo adaptándose a lo que puede encontrar (Chuang, Cubbin, Ahn, y Winkleby, 2005).

Las interacciones entre los tres factores de la regulación, la "disponibilidad de sustitutos" (reforzadores alternativos), el "rango de precios" (valor de las unidades estándar de refuerzo), y el "nivel de ingresos" (disponibilidad de repertorio o tiempo para emplearlo), son diversas. Por ejemplo, Epstein, Leddy, Temple y Faith (2007) reseñan un experimento de elección entre alimentos poco sanos adversus alimentos saludables, en el cual niños de 10 a 14 años acostumbrados a la "comida chatarra" pasaron por diferentes niveles de ingreso: "bajo", "regular" y "alto". Cuando los niños estaban en la condición de "bajo ingreso" aumentaron su consumo de alimentos saludables (tales como frutas o yogurt), pero cuando estuvieron en el nivel alto siguieron eligiendo los productos poco sanos, aunque el precio de estos aumentara. En consecuencia, puede esperarse que la elección de "sustitutos" se reduzca cuando el individuo goce de mejor status económico. No obstante, también se ha podido advertir que el factor de disminución del precio de las comidas sanas incrementa su probabilidad de elección, incluso más que el factor de propaganda a favor de hábitos saludables. En general, una evaluación de las preferencias que los individuos tienen y sus determinantes pueden proporcionar información adecuada acerca de la sensibilidad a los aumentos de precios. Los resultados sugieren que la determinación de cuán sensible es la demanda de un individuo para un refuerzo (es decir, la cuantificación de la elasticidad de la demanda) puede ayudar al analista de conducta a predecir qué consecuencias podrían producir una mayor persistencia de la respuesta en el ámbito aplicado.

\section{Decisiones de perspectiva y decisiones clínicamente significativas}

Como se ha visto, las investigaciones conductuales abordan problemas muy complejos de redistribución de respuestas, debido a que los comportamientos de consumo y empresarial comprenden acciones involucradas con contingencias de selección, adquisición o utilización de bienes, servicios, ideas o experiencias para obtener beneficios de diverso 
tipo (llámese satisfacer sus "necesidades" y "deseos"). Todo eso supone procesos de decisión que preceden y son consecuencia de tales acciones (Rachlin, 1995). El método de investigación más común en la teoría de las perspectivas es poner a los participantes en situación de elegir entre dos hipotéticos resultados futuros (por ejemplo, a elegir entre una cosa segura y un resultado de riesgo. Los datos, consignados a través del autoinforme de los sujetos, son sometidos a análisis estadísticos obteniendo el promedio de elecciones de los individuos y verificando su significatividad. Según los datos aportados por estas indagaciones (Kahneman \& Tversky, 1984), incluso en el mejor de los casos, las personas acostumbran emitir juicios probabilitarios inexactos, basándose sólo en la semejanza de una información concreta con el prototipo ideal.

Aunque ha sufrido críticas por la aparente subjetividad de los datos que brinda, este método de autoinforme tiene valor en ciertas circunstancias, pues hay un número muy grande de decisiones importantes tomadas por los individuos que podrían calificarse de "opciones de perspectiva", donde ellos sopesan resultados posibles antes de haberlos experimentado. Hay, sin embargo, otras decisiones que involucran factores clínicamente significativos porque son patrones repetidos hasta el extremo de la "autodestrucción" — por ejemplo, el abuso de sustancias psicoactivas y el juego patológico-, afectando el estado de salud. En dichos casos se hace necesario indagar cómo las experiencias anteriores del individuo respecto a las consecuencias que han afectado históricamente su conducta, afectan sus actuales respuestas prospectivas. Es evidente que cada cual suele comportarse ante nuevas opciones como hizo en el pasado si ese patrón de elección ha sido seleccionado por las consecuencias de la ejecutoria habitual (Francisco et al., 2009).

Una forma de acercamiento entre la teoría de la perspectiva y el análisis conductual es la explicación del porqué los individuos acostumbran decidir sobre sus asuntos importantes de maneras no racionales (sin calcular probabilidades), en función a estrategias informales adoptadas en "entornos de incertidumbre", es decir, ambiguos y poco claros. Kahneman (2003) intenta explicar ese efecto mediante la postulación de categorías que denomina "atajos heurísticos", quizá basados en repertorios genéticamente adaptativos. La toma de un atajo heurístico muy común es el que se guía por la aversión a la pérdida: se teme más lo que se puede perder que lo que se puede ganar tomando la decisión. En términos técnicos conductuales se hablaría de respuestas implicadas en contextos de contingencias abiertas que han sido seleccionadas por consecuencias aversivas, las cuales aumentaron la sensibilidad del comportamiento a las pérdidas potenciales y la intolerancia al retraso de la gratificación reforzadora. Sobre este repertorio extrañamente (in)adaptativo, Magoon y Critchfield (2008) discuten si se trata de una tendencia filogenética evolutivamente desarrollada en: a) un ambiente natural lleno de depredadores, b) de competencia entre miembros de la propia especie, c) de suministros de alimentos impredecibles, y d) de escasas oportunidades de apareamiento; condiciones que pueden resultar afectadas por una historia ontogenética de experiencias. Esto parece ser todavía un enigma que, sin duda, los próximos estudios experimentales de la economía conductual tendrán que intentar resolver. 


\section{CONCLUSIÓN}

Los análisis conductuales básicos del comportamiento de consumo hechos a partir de la experimentación de laboratorio, y donde el papel de la economía de fichas así como también de la hipótesis de la regulación conductual fueron y son relevantes, resultan de gran utilidad conceptual para evaluar innumerables situaciones, por ejemplo de salud (digamos el uso de drogas o la alimentación sana, ver la compilación de Bickel y Vusinich, 2000), de elección de candidatos políticos, de relaciones interpersonales, de elecciones vocacionales, etcétera. Los procesos decisorios se han mostrado como influidos por las restricciones a que está sujeto el comportamiento del consumidor. En la traducción de términos económicos a experimentales, esto es: a) la "disponibilidad de sustitutos" dada por la posibilidad de contar con reforzadores alternativos, b) el "rango de precios" del producto, o valor de las unidades estándar de refuerzo, y c) el "nivel de ingresos" o disponibilidad de repertorio o tiempo para emplearlo en busca del reforzador.

Lo cierto es que en esos casos, las respuestas en contextos contingenciales diversos son normalmente seleccionadas por sus consecuencias. En contextos más inciertos como son los de "incertidumbre", para explicar el comportamiento de elección entran a tallar variables más hipotéticas que parecieran fruto de una combinación de factores filogenéticos y ontogenéticos, pero siempre relacionados con la historia de reforzamiento del organismo a largo plazo.

\section{REFERENCIAS BIBLIOGRÁFICAS}

1. Alejo, A.; Rojas, P. y Pérez-Acosta, A. (2008). Psicología y asuntos económicos: Una aproximación al estado del arte. International Journal of Psychological Research, 1(1), 49-57. Disponible en: http://mvint.usbmed.edu.co:8002/ojs/index.php/web/ article/viewPDFInterstitial/413/404

2. Allison, J. (1993). Response deprivation, reinforcement, and economics. Journal of Experimental Analysis of Behavior, 60, 129-140. Disponible en: http://www.ncbi. nlm.nih.gov/pmc/articles/PMC1322150/pdf/jeabehav00003-0131.pdf

3. Ayllon T, Azrin N.H. (1965). The measurement and reinforcement of behavior of psychotics. Journal of Experimental Analysis of Behavior; 8(6):357-383. Disponible en: http://www.ncbi.nlm.nih.gov/pmc/articles/PMC1338117/

4. Bentham, J. (1978). Escritos económicos. México: Fondo de Cultura Económica.

5. Bickel, W.K. y Vuchinich, R.E. (Eds.) (2000). Reframing health behavior: Change with behavioral economics. New Jersey: Lawrence Erlbaum Associates.

6. Bickel, W.K. Green, L. y Vuchinich, R.E. (1995). Behavioral economics. Journal of Experimental Analysis of Behavior, 64, 257-262. Disponible en: http://www.ncbi. nlm.nih.gov/pmc/articles/PMC1350136/pdf/jeabehav00216-0007.pdf

7. Chuang, Y., Cubbin, C., Ahn, D. y Winkleby, M.A. (2005). Effects of neighbourhood socioeconomic status and convenience store concentration on individual level smoking. 
Journal of Epidemiology and Community Health, 59, 568-573. Disponible en: http:// www.jstor.org/pss/25570774

8. DeGrandpre, R.J., Bickel, W.K., Rizvi, S.A. y Hughes, J.R. (1994). A behavioral economic analysis of concurrently available money and cigarettes. Journal of Experimental Analysis of Behavior. 61(2): 191-201. Disponible en: http://www. ncbi.nlm.nih.gov/pmc/articles/PMC1334407/

9. Denegri, M. (2004/2010). Introducción a la psicología económica, Edición electrónica. Disponible en: www.eumed.net/libros/2010b/681/

10. Domjan, M. (2007/2010). Principios de aprendizaje y conducta. México: Cengage Learning Editores. Disponible en: http://www.4shared.com/document/w6sC2Hxy/ Principios_de_aprendizaje_y_co.html

11. Epstein, L.H.; Leddy, J.J.; Temple, J. y Faith, M.S. (2007). Food reinforcement and eating: A multilevel análisis. Psychological Bulletin, 133, 884-906. Disponible en: http://www.ncbi.nlm.nih.gov/pmc/articles/PMC2219695/

12. Fisher, E.B., Winkler, R.C.; Krasner, L.; Kagel, L.J.; Battaliod, R.C. \& Basmann, R.L. (1978). Economic perspectives in behavior therapy: Complex interdependencies in token economies. Behavior Therapy, 9(3), 391-403. Disponible en: http://www.sciencedirect.com/science?_ob=ArticleURL\&_udi=B7XMW4 K605J7-9\&_user $=10 \&$ \& coverDate $=06 \% 2$ F $30 \% 2$ F $1978 \&$ rdoc $=1 \&$ $\mathrm{fmt}=$ high \&_orig $=$ search $\&$ _origin $=$ search $\&$ _sort $=\mathrm{d} \&$ _docanchor $=\& \mathrm{view}=\mathrm{c} \&$ _ 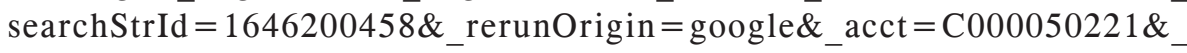 version $=1 \&$ _urlVersion $=0 \&$ userid $=10 \& \mathrm{md} 5=\mathrm{d} 2 \mathrm{~b} 6 \mathrm{df} 4 \mathrm{~b} 55524 \mathrm{a} 8260 \mathrm{~cd} 4 \mathrm{ce} 47 \mathrm{df} 4$ d189\&searchtype $=\mathrm{a}$

13. Francisco, M.T., Madden, G.J., y Borrero, J. (2009). Behavioral economics: principles, procedures, and utility for applied behavior analysis. The Behavior Analyst Today, 10, 277-294. Disponible en: http://www.thefreelibrary.com/Behavioral + economics \%3A + principles, + procedures, + and + utility + for + applied...-a0214102537

14. Hursh, S.R., \& Silberberg, A. (2008). Economic demand and essential value Psychological Review, 115, 186-198.

15. Kagel, J.H. y Winkler, R.C. (1972). Behavioral economics: areas of cooperative research between economics and applied behavioral analysis. Journal of Applied Behavior Analysis, 5(3):335-342. Disponible en: http://www.ncbi.nlm.nih.gov/ pmc/articles/PMC1310770/

16. Kahneman, D., \& Tversky, A. (1984). Choices, values, and frames. American Psychologist, 39, 341-350. Disponible en: http://www.mudancasclimaticas.andi.org.br/ download.php?path $=1$ heobe896kls8t7vab26.pdf

17. Kahneman D. (2003). Mapas de racionalidad limitada: Psicología para una economía conductual. Revista Asturiana de Economía, 28, 181-225.

18. Magoon, M.A., \& Critchfield, T.S. (2008). Concurrent schedules of positive and negative reinforcement: Differential-impact and differential-outcomes hypotheses. 
Journal of the Experimental Analysis of Behavior, 90, 1-22. Disponible en: http:// seab.envmed.rochester.edu/jeab/articles/2008/jeab-90-01-0001.pdf

19. Mayorga, P.; Albañil, N. y Cómbita, L. (2006). Elección y sustitución de estímulos entre el alcohol y el alimento en ratas: una explicación del consumo de drogas desde el contexto de la economía conductual. Suma Psicológica, 13(1), 33-50. Disponible en: http://pepsic.bvsalud.org/pdf/suma/v13n1/v13n1a04.pdf

20. Murphy, J. Correla, C. y Barnett, N. (2007). Behavioral economic approaches to reduce college student drinking. Addictive Behaviors, 32, 2573-2585.

21. Phillips E.L., Phillips E.A, Fixsen D.L. y Wolf MM. (1971). Achievement Place: modification of the behaviors of pre-delinquent boys within a token economy. Journal of Applied Behavior Analysis; 4(1):45-59. Disponible en: http://www.ncbi.nlm.nih. gov/pmc/articles/PMC1310667/

22. Sapford, D., Phythian-Adams, S.L. y Apps, E. (2009). Behavioural economics. A review of the literature and proposals for further research in the context of workplace health and safety. Liverpool: University of Liverpool. Disponible en: http://www. hse.gov.uk/research/rrpdf/rr752.pdf

23. Silberberg, A., Warren-Boulton, F. R. y Asano, T. (1987). Inferior-good and Giffengood effects in monkey choice behavior. Journal of Experimental Psychology: Animal Behavior Processes, 13(3), 292-301.

24. Staats, A. W. y Staats, C.K.(1963). Complex Human Behavior. A systematic extension of learning principles. New York: Holt, Rinehart and Winston.

25. Staats, A.W. (1975/1979). Conductismo Social. México: El Manual Moderno,

26. Staats, A.W., Staats, C. K., Schutz, R. E. y Wolf, M. (1962). The conditioning of reading responses using "extrinsic" reinforcers. Journal of Experimental Analysis of Behavior, 5, 33-40. Disponible en: http://www.ncbi.nlm.nih.gov/pmc/articles/ PMC1404175/

27. Sumpter, C.E., Temple, W. \& Foster, T.M. (2004). Comparing demand functions when different price manipulations are used: Does unit price help? Learning \& Behavior, 32 (2), 202-212. Abstract Disponible en: http://www.ncbi.nlm.nih.gov/ pubmed/15281392

28. Rachlin, H. (1995). Behavioral economics without anomalies, Journal of Experimental Analysis of Behavior, 64, 397-404. Abstract Disponible en: http://www.ncbi.nlm. nih.gov/pubmed/8551195

29. Vieira, M. (2008). Nuevos paradigmas del pensamiento económico: Un llamado a la interdisciplinariedad. Revista Ciencias Estratégicas, 16(20), 335-350. Disponible en: http://www.dialnet.unirioja.es/servlet/dcfichero articulo? codigo $=2991267 \&$ orden $=0$

30. Winkler, R.C. (1971). The relevance of economic theory and technology to token reinforcement systems. Behavioral Research Therapy, 9(2):81-88. Disponible en: http://www.ncbi.nlm.nih.gov/pubmed/5556841 